\title{
Baroclinic Motions and Energetics as Measured by Altimeters
}

\author{
CARL WUNSCH \\ Department of Earth, Atmospheric and Planetary Sciences, Massachusetts Institute of Technology, Cambridge, Massachusetts
}

(Manuscript received 24 February 2012, in final form 5 July 2012)

\begin{abstract}
Small surface displacements appearing in tide gauge and altimetric records are used to detect hydrostatic baroclinic modes in the ocean. Those deflections are a small fraction of the interior isopycnal vertical displacements and are dependent directly upon the in situ stratification. Conversion of surface height to interior amplitudes and energies encounters significant spatial and seasonal shifts that need to be accounted for in quantitative use. This technical article analyzes the global-scale spatial variations in the relationship between surface deflections and interior motions. Similar considerations make it possible to use altimetric data to estimate the deep interior temperature variability as a function of position, calculations having a strong influence on abyssal trend determination in the presence of eddies.
\end{abstract}

\section{Introduction}

Altimetric measurements of baroclinic motions have grown increasingly important over the past almost 20 years. Of particular interest have been studies of the internal tides as seen in the Ocean Topography Experiment (TOPEX)/Poseidon-Jason series satellites (Ray and Mitchum 1997, and many succeeding papers) but also including geostrophic-balanced motions (e.g., Wunsch 1997; Xu et al. 2011), and the recent interest in so-called surface quasigeostrophic (SQG) modes (LaCasce and Mahadevan 2006; Isern-Fontanet et al. 2008).

The purpose of this article is to call attention to some of the technical details of the way in which internal baroclinic motions become visible from space, and to suggest a bit of caution in the quantitative estimates being made of, for example, energy gains and losses in propagating modes. Although the dynamics are simple, one is in the situation in which the entirety of an important observational system rests upon a very small physical effect—one that is commonly suppressed as being negligible. The phenomenon was exploited by Wunsch and Gill (1976, hereafter WG) to demonstrate the existence of equatorially trapped waves in tide gauge data. Rainville and Pinkel (2006) discuss many of the same issues in the context of the internal tide, but similar

Corresponding author address: Carl Wunsch, Department of Earth, Atmospheric and Planetary Sciences, Massachusetts Institute of Technology, Cambridge, MA 02139.

E-mail: cwunsch@mit.edu considerations apply generally to wavelike motions observed in tide gauges and altimeters. Thus the greater emphasis here is on balanced motions and on the possible pitfalls of inferring large interior vertical excursions from truly minor surface expressions. Because the dynamics are elementary, but detailed, much of the development here is given in an appendix, with only a summary in the main body of the present paper.

\section{Some elementary theory}

Textbooks show how for periodic, linear, Boussinesq, hydrostatic limit motions and horizontally uniform stratification, an assumption of separation of variables

$$
\begin{aligned}
{[u(x, y, z, \sigma), v(x, y, z, \sigma)] } & =[U(x, y, \sigma), V(x, y, \sigma)] F(z) \\
w(x, y, z, \sigma) & =P(x, y, \sigma) G(z) \\
p^{\prime}(x, y, z, \sigma) & =\rho_{0} P(x, y, \sigma) F(z) \\
\rho^{\prime}(x, y, z, \sigma) & =-\frac{\rho_{0} N(z)^{2}}{i \sigma g} P(x, y, \sigma) G(z)
\end{aligned}
$$

leads in a resting, unforced ocean of mean density $\rho_{0}$ to two equivalent differential equations,

$$
\frac{d^{2} G(z)}{d z^{2}}+N^{2}(z) \gamma^{2} G(z)=0
$$




$$
\frac{d}{d z}\left[\frac{1}{N^{2}(z)} \frac{d F}{d z}\right]+\gamma^{2} F(z)=0,
$$

subject to boundary conditions. Here $N(z)$ is the buoyancy frequency, $p^{\prime}$ is the pressure perturbation about a resting hydrostatic state, $\sigma$ is the radian frequency, and $\gamma^{2}$ is a separation constant that must be determined. Horizontal structures in $U, V$, and $P$ are determined from horizontal partial differential operators

$$
\mathcal{L}_{q}(q, x, y, \sigma, \gamma)=0
$$

where $q$ is any of the dependent variables, $u, v, w, p$, or $\rho^{\prime}$, and the corresponding $\mathcal{L}_{q}$ in various limits describe Rossby, equatorial, internal, Kelvin, and inertial waves. In the hydrostatic limit used here, the vertical structures are, in the unforced problem, independent of which of these physical situations one is describing. Spherical coordinates can be used in $\mathcal{L}_{q}$ if appropriate.

If the motion is periodic, with all variables proportional to $\exp (-i \sigma t), F$ and $G$ are connected by

$$
\begin{aligned}
\frac{i \sigma}{N^{2}(z)} \frac{d F}{d z} & =G(z), \\
\frac{d G(z)}{d z} & =-i \sigma \gamma^{2} F(z) .
\end{aligned}
$$

and the references show that at the free surface, the linearized dynamical boundary conditions are

$$
g \gamma^{2} G(0)-\frac{d G(0)}{d z}=0
$$

or

$$
F(0)+\frac{g}{N^{2}(0)} \frac{d F(0)}{d z}=0
$$

and at a rigid bottom, either

$$
G(-h)=0,
$$

if using $G$, or

$$
\frac{d F(-h)}{d z}=0 .
$$

Depending upon the particular problem, the separation constant (as well as $F, G$ ) can be determined either by Eqs. (2) or (3), and (6) or (7), as a vertical eigenvalue (Sturm-Liouville) problem, or from the horizontal problem (4). In practice, the vertical deflection $G_{D}(z)=G(z) /(-i \sigma)$ is often more convenient. Let $z_{\max }$ be the depth of the maximum of $\left|G_{D}(z)\right|$ over the water column, and set $\left|G_{D}\left(z_{\text {max }}\right)\right|=1$, so that the dimensional vertical deflection is $B G_{D}(z)$, where $B$ is a constant.

Most authors analyzing altimetric data have used some form of Eq. (2) or (3) with realistic estimates of $N$ $(z)$, but have commonly employed a rigid-lid boundary condition at $z=0$ instead of the dynamic boundary condition of a free surface (e.g., Zhao et al. 2010). Thus, (6) would become $G(0)=0$, and (7) is then $d F(0) / d z=0$. The question raised here is whether this simplification, which is known to be an excellent one and has no significant influence on the dynamics (e.g., Pedlosky 1987, p. 367), is of any importance to the observations that are wholly dependent upon failure of the rigid-lid approximation. ${ }^{1}$

\section{a. Constant $N$}

The simplest case is that of $N=N_{0}=$ constant and is worked out in numerous textbooks for a free surface. One finds the familiar requirement

$$
\tan \left(N_{0} \gamma h\right)=\frac{N_{0}}{g \gamma},
$$

with multiple roots, $\gamma_{j}, j=0,1, \ldots$ Excluding the lowest root, $\gamma_{0} \approx 1 / \sqrt{g h}$, corresponding to the barotropic mode, $\gamma_{j}=\pi j / N_{0} h+\Delta_{j}$, and where $\Delta_{j} \approx N_{0} / g \pi j \ll \pi j / N_{0} h$. (A more physically immediate variable is the "equivalent depth," $h_{j}^{\prime}=1 / g \gamma_{j}^{2}$, having units of length, so that $h_{j}^{\prime} \approx N_{0}^{2} h^{2} /\left(g j^{2} \pi^{2}\right) \ll h$, and is discussed below. $)$ Then,

$$
\begin{aligned}
G_{\mathrm{D} 1}(z) & =B \sin \left[\left(\frac{\pi}{h}+\frac{N_{0}^{2}}{g \pi}\right)(z+h)\right] \\
& =B \sin \left[\left(\frac{\pi}{h}+\frac{N_{0}^{2}}{g \pi}\right)(z+h)\right]
\end{aligned}
$$

and

$$
F_{1}(z)=\frac{N_{0}^{2} h}{g \pi} B \cos \left[\left(\frac{\pi}{h}+\frac{N_{0}^{2}}{g \pi}\right)(z+h)\right],
$$

the latter having a zero crossing in the middle of the water column where $G_{1}$ is a maximum.

$$
\text { At } z=0 \text {, }
$$

$$
G_{\mathrm{D} 1}(0)=-B \sin \left(\Delta_{1}\right) \approx-B \frac{N_{0}^{2} h}{g \pi} \neq 0 .
$$

\footnotetext{
${ }^{1}$ With the rigid lid, the barotropic mode is calculated separately. The approximation is required also, for instance, to make the inference, $\int_{-h}^{0} F(z) d z=0$, identically, for baroclinic modes, a good but not perfect equality.
} 
Notice the minus sign. For higher roots the corresponding $G_{\mathrm{Dj}}(0)$ are proportionally reduced. In the rigid-lid approximation, this value is identically zero. The numerical value of the surface displacement, $\eta=-B N_{0}^{2} h / g \pi$, is sensitive to the choice of $N_{0}$. Inversely, if one is attempting to deduce the interior vertical amplitude, the amplification factor from $\eta$ is $B=-g \pi \eta / N_{0}^{2} h$. With $N_{0}=3$ cycles per hour (cph), and $h=4000 \mathrm{~m}$, then $\gamma=0.15$, $\eta \approx-3.5 \times 10^{-3} \mathrm{~m}$, and $h_{1}^{\prime} \approx 4 \mathrm{~m}$, which would translate into about a $3.5-\mathrm{cm}$ surface depression for $B=$ $10 \mathrm{~m}$. The value applies both to balanced and internal waves.

A 1-m interior vertical displacement of an isopycnal generates only a few millimeters of surface elevation change. To the extent that the elevation signal is to be used to infer propagation losses and gains in the interior motions, the potential arises for sizeable errors, particularly in quadratic quantities such as energies. If $N(z)$ and $h$ were laterally constant, then comparisons of relative amplitudes and energies would be simple, and independent of exactly how the sea surface condition was employed. Thus global spatial maps of the relationship between interior and surface deflections become of interest. See Rainville and Pinkel (2006) for discussion of the Wentzel-Kramers-BrillouinJeffreys (WKBJ) approximation specifically for internal tides.

\section{b. Compensation}

A physical interpretation is helpful. If $w>0$ within the water column, the perturbation increases the density at all depths, surface elevation $\eta<0$ and vice versa-that is, the vertical displacement overshoots 0 at the free sea surface, producing a $180^{\circ}$ phase shift from the interior vertical motion. The overshoot is readily understood as providing, in this hydrostatic limit, that amount of pressure anomaly from the elevation to just compensate the density anomaly generated in the interior, so as to produce a 0 pressure gradient and hence a "level of no horizontal motion" at the first 0 crossing of $F_{1}(z)$. This 0 level is maintained throughout the entire wave period as $w$ reverses sign. (It is a level of maximum vertical motion.)

Interpretation of the surface deflection is thus the one conventionally associated with the dynamic method: upwelling of cold water corresponds there to a negative surface anomaly and downwelling of warm water to a positive surface elevation, with magnitudes consistent with a supposed level of no motion. Here such a level does exist in the wave motion, and is required by the dynamics. The magnitude of $\eta$ is diminished for the higher modes because they produce levels of no horizontal motion ever closer to $z=0$, hence generating smaller density anomalies needing compensation for fixed maximum vertical displacements.

\section{c. Comparison to rigid-lid approximations}

A rigid-lid condition can be imposed for all solutions by demanding $G(0)=0$ instead of the pressure continuity condition there. For small $\gamma$, such a solution violates the dynamic free surface boundary condition, but has little influence on the vertical structures except right at the sea surface. A conventional approach to employing the approximation of a rigid lid is to calculate the elevation $\eta_{\mathrm{RL}}$ corresponding to the pressure exerted on that hypothetical surface. Thus, in the present case,

$$
p_{\mathrm{RL}}^{\prime}(0)=-\left.\frac{\rho_{0} N_{0}^{2} h}{\pi} B \cos \left(\frac{\pi z}{h}\right)\right|_{z=0}=\rho_{0} g \eta_{\mathrm{RL}}
$$

and

$$
\eta_{\mathrm{RL}}=-\frac{N_{0}^{2} h}{g \pi} B
$$

The full boundary condition produces [Eq. (12)]

$$
\eta=-\frac{N_{0}^{2} h}{g \pi} B \cos \left(\frac{N_{0}^{2} h}{g \pi}\right),
$$

and which to order $\left(N_{0}^{2} h / g \pi\right)^{2}$ is identical. Thus, in this case the rigid-lid boundary condition approximation is a good one. Unfortunately, it need not be so with realistic profiles. The appendix compares the elevations for a rigid lid and the free surface boundary condition, using an exponential $N(z)$ profile. Factor of 2 differences are found as a function of $N(0)$. Chiswell (2006) found factor of 2 discrepancies between altimetric and mooring amplitudes-roughly consistent with the analytical result—although he offers different explanations.

In some theories of propagation over topography (e.g., Bobrovich and Reznik, 1999) and in observations (Wortham, 2012), zero bottom pressure variation appears to be a better approximation (a bottom level of no motion), and which is equivalent to postulating coupling of the barotropic and baroclinic mode solutions and thus is not a consequence of the Sturm-Liouville problem.

The behavior is easily analyzed for the $N=$ constant case. With a rigid lid, $F_{1}(z=-h)=\cos (\pi)$, and forcing a zero-bottom-pressure-perturbation doubles the surface elevation from the basic solution. Bottom pressure can be additive or subtractive to the surface pressure, depending upon whether the mode number is even or odd. For more complex profiles, various surface amplitude possibilities emerge. 

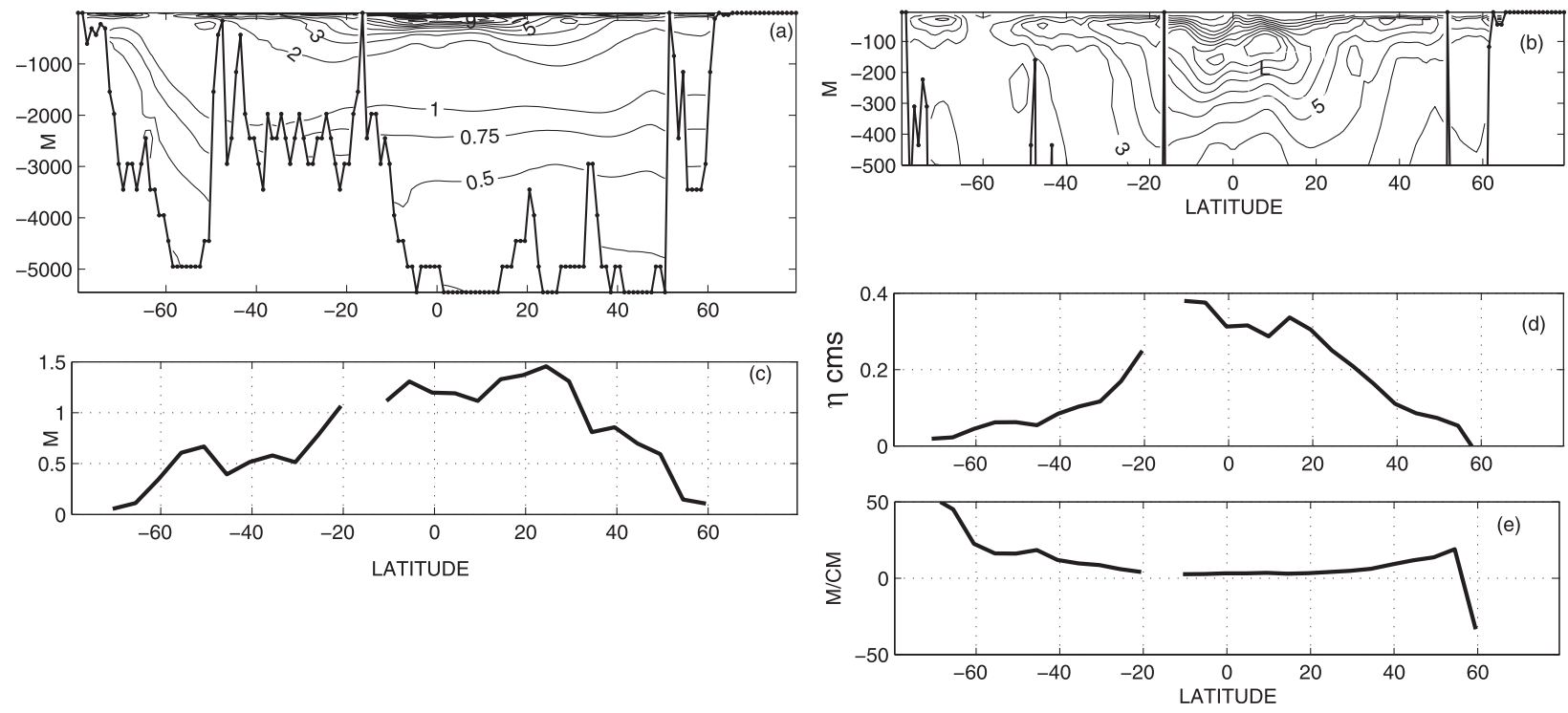

FIG. 1. (a) Buoyancy frequency $N(z)$ along the meridian $179.5^{\circ} \mathrm{E}$ in cycles per hour (cph). (b) An expanded view of the top $500 \mathrm{~m}$. Maximum value is $11 \mathrm{cph}$ and minimum is $0.09 \mathrm{cph}$. Vertical average values diminish toward high latitudes. In the deep Southern Ocean, however, the abyssal stratification increases poleward. (c),(d) Values of $h_{1}^{\prime}$ and $\eta$, respectively, as a function of latitude along $180^{\circ}$ longitude. Note that $\eta$ can be interpreted as $\mathrm{cm} \mathrm{m}^{-1}$ of interior vertical displacement. (e) Values of $1 / \eta$, the amplification factor for converting from $\eta$ in centimeters into the maximum interior vertical displacement in meters. The gap corresponds to the arcs associated with Samoa and other island groups to the west in Fig. 2.

\section{d. Other analytical profiles}

Analytical (closed form) solutions exist to Eqs. (2) and (3) subject to various boundary conditions, including constant $N$ underlying a mixed layer $(N=0)$ and the exponential profile, as well as others. These are briefly explored in the appendix, but to keep this note as short as possible, attention is now turned to realistic profiles, $N(z)$.

\section{Observed $N(z)$}

The $N(z)$ used is taken from Estimating the Circulation and Climate of the Ocean (ECCO) family of solutions, version 3.73 (e.g., Wunsch et al. 2009), and is thus a 16-yr average of a model fit by least squares to in situ temperature and salinity plus a large variety of other (e.g., satellite) data. A section for $N(z)$ is displayed in Fig. 1 along the meridian $180^{\circ}$, and Fig. 2 displays horizontal maps of the estimated values. The horizontal spatial gradients are of particular concern for comparing propagation losses and gains. To the extent that changes are abrupt relative to wavelengths, conventional WKBJlike approximations will fail, although those phenomena are not taken up here (see, e.g., Rainville and Pinkel 2006). However, wavelengths on the order of $100 \mathrm{~km}$ come uncomfortably close to lateral distances over which $N(z)$ changes significantly, and the use of separation of variables methods must be reexamined. Topographic changes are even more rapid and the combination shows up through the mapped values of $h_{i}^{\prime}$. Thus, depending upon the analyst's purposes, many useful approximations, generically related to index of refraction changes, can become quantitatively doubtful. Present model resolution cannot show variations below a $100-\mathrm{km}$ scale.

Equations (5a) and (5b) readily lend themselves to numerical solution by relaxation, subject here to the three boundary conditions, $G(-h)=0, d F(-h) / d z=0$, and $F(0)+G(0) /(-i \sigma)=0$, because $\gamma$ is unknown. Figure 3 shows the $34.5^{\circ} \mathrm{N}$ forms of $F_{1}$ and $G_{\mathrm{D} 1}$ and in which the near-surface intensification of $F_{1}$ is particularly striking as well as a less extreme case in the Southern Ocean.

Values of $\gamma_{1}, \eta$, and the amplification factor $1 / \eta$ along the $180^{\circ}$ meridian are shown in Fig. 1 . The separation constant $\gamma_{1}^{2}$ varies on a global scale. It proves physically more meaningful to map instead the equivalent depth, $h_{1}^{\prime}=1 / g \gamma_{1}^{2}$, displayed in Fig. 4 as computed every $5^{\circ}$ of latitude and longitude. Equivalent depths control the horizontal phase velocities of the corresponding modes in the familiar combination, $\sqrt{g h_{1}^{\prime}}$, as well as the Rossby radius of deformation, and are thus an important element of descriptive oceanography. The value of $h_{1}^{\prime}$ is generally on the order of $1 \mathrm{~m}$ except at higher latitudes where it becomes very small, corresponding there to the very slow propagation velocity of linear baroclinic motions. Figure 5 shows the qualitatively important spatial changes in 

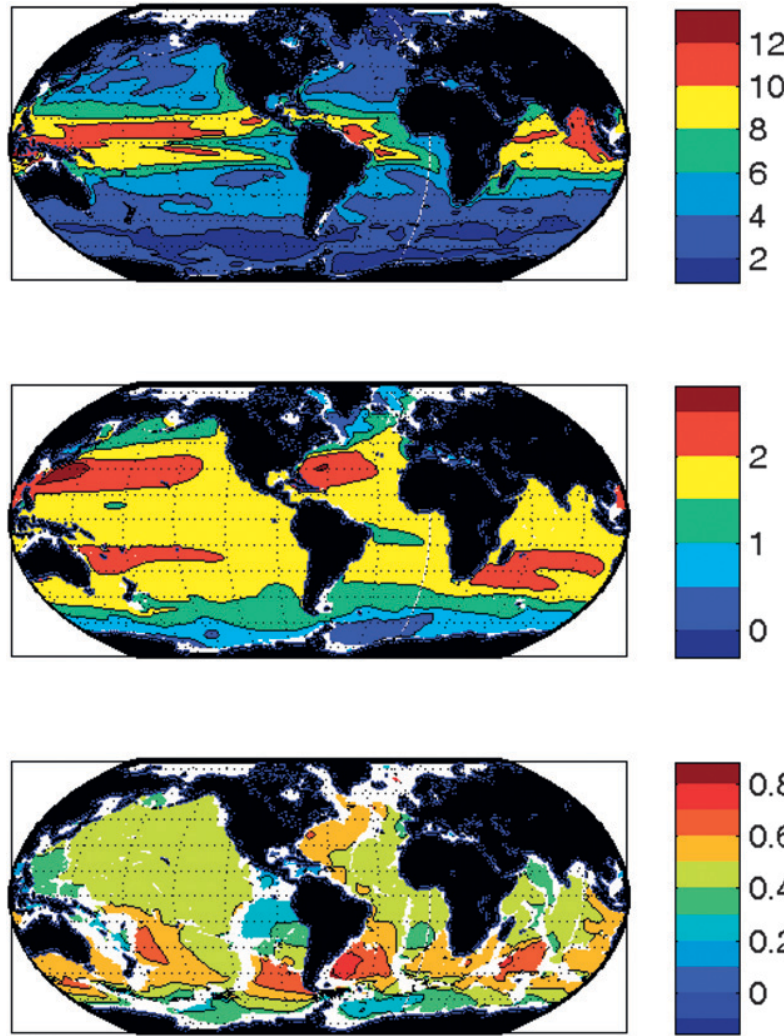

FIG. 2. Buoyancy frequency (cph) from the ECCO estimate (top to bottom) at $117.5,847.5$, and $3450.5 \mathrm{~m}$. Considerable lateral heterogeneity is apparent.

surface displacement $\eta$ in centimeters per $10 \mathrm{~m}$ of interior vertical isopycnal displacement amplitude.

Its reciprocal—the amplifying factor (not shown)—has, in the Pacific Ocean, a comparatively simple, mainly zonal, structure. That ocean is often used for altimetric studies because of its great expanse. Meridional gradients at mid and low latitudes, although not qualitatively great, are large enough to generate systematic, quantitative changes with position (e.g., as one moves away from the Hawaiian Islands). Strong zonal gradients exist in the midlatitude Atlantic Ocean and which would raise questions about the interpretation, for example, of wavenumber spectra or energy propagation effects not explicitly dealt with here.

Amplification relative to the interior values of the nearsurface horizontal currents is a near-universal character of the global modes, but its degree does vary with location, as can be seen in Fig. 6 displaying $\left|F_{1}(0) / F_{1}(-h)\right|$. Because pressure perturbations are also proportional to $F_{1}(z)$, Fig. 6 depicts the relationship between bottom pressure and that seen at $z=0$. Similarly, the depth $z_{\max }$ tends to lie between about 1000 and $1500 \mathrm{~m}$ (not shown), but with considerable expanse of exceptional regions at high latitudes. Note that $\left|B G_{\mathrm{D} 1}\left(z_{\max }\right)\right|$ is a measure of the mesoscale signal induced in the deep and abyssal ocean by variability visible at the surface. That in turn has consequences for the ability to infer climate time scale trends from sparse hydrographic samples. Temperature disturbances implied by these modes, in the linearized limit, are of the form

$$
\Delta T(z)=\frac{\partial T}{\partial z} G_{\mathrm{D} 1}(z)
$$

per unit displacement, where $\partial T / \partial z$ is the adiabatic temperature gradient. The vertical displacement at $z_{\max }$ can be estimated by using the RMS value of $\eta$ obtained
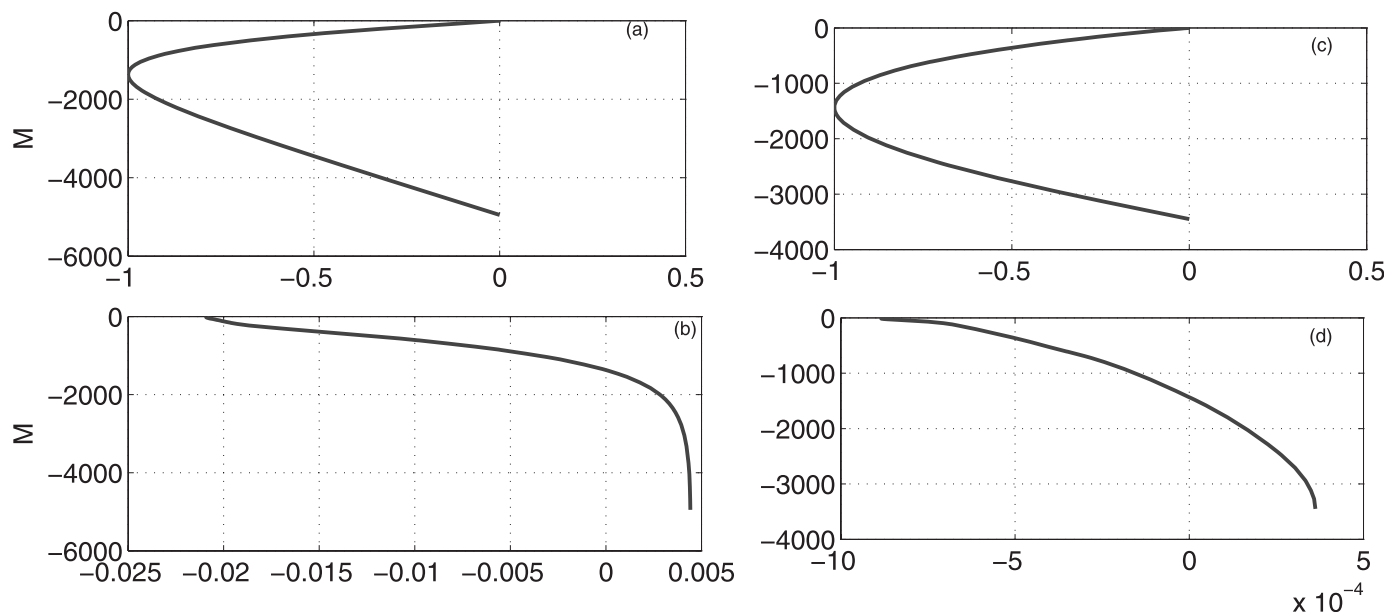

FIG. 3. Values of (a) $G_{1}$ and (b) $F_{1}$ for the realistic $N(z)$ at $34.5^{\circ} \mathrm{N}, 180^{\circ} \mathrm{E}$. Note the very strong near-surface amplification in $F_{1}$ relative to its bottom value. This behavior is almost universal, but the degree of amplification varies considerably as can be seen in the right panels. (c),(d) As in (a),(b), but for the Southern Ocean at $65.5^{\circ} \mathrm{S}, 4.5^{\circ} \mathrm{E}$ (note change in depth). 


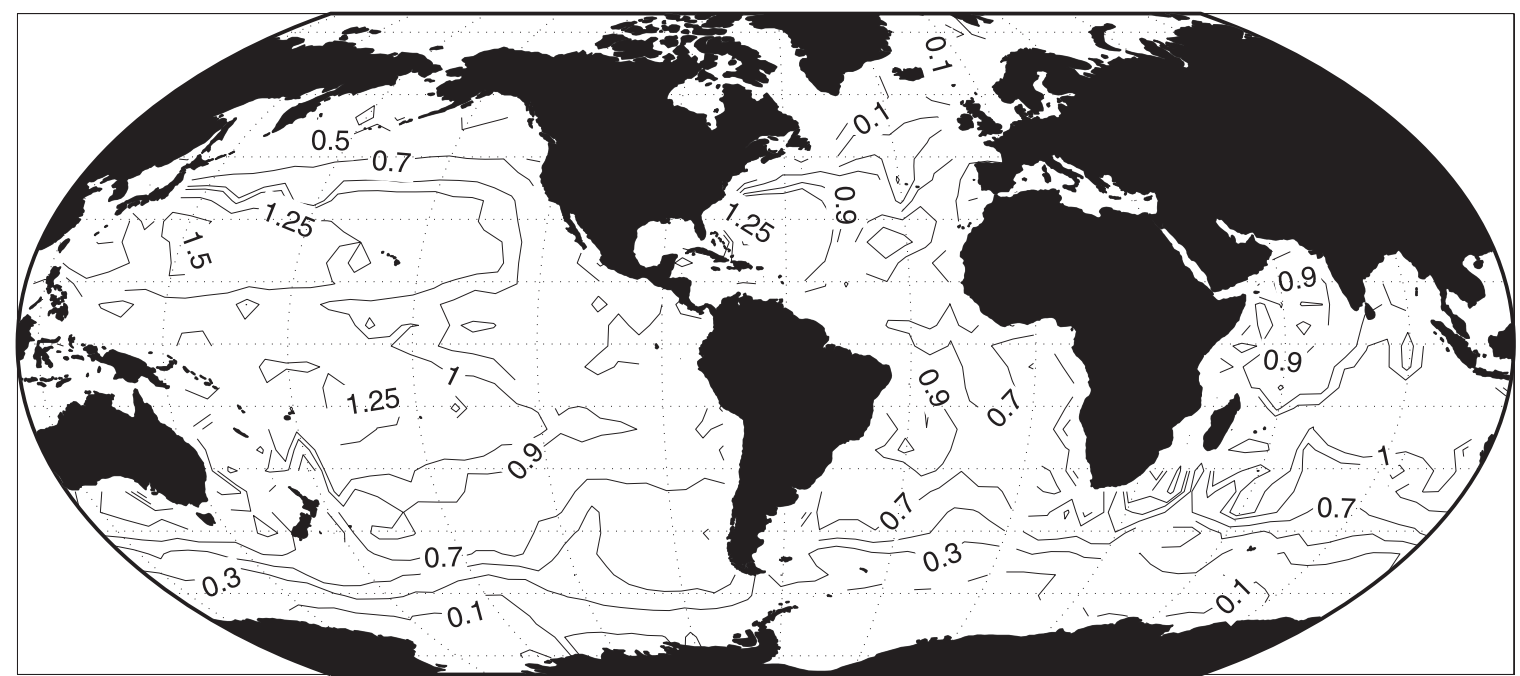

FIG. 4. Equivalent depth $h_{1}^{\prime}=1 / g \gamma_{1}^{2}(\mathrm{~m})$, which depends upon both $N(z)$ and $h$. Values vary from greater than $1 \mathrm{~m}$ at low latitudes to about $10 \mathrm{~cm}$ in subpolar regions. Note that the Rossby radii are readily computed from $R_{j}=\sqrt{g h_{j}^{\prime}} / f$ (not shown). Compare to the map of $M_{2}$ tide group velocity in Rainville and Pinkel (2006).

from altimetry (e.g., Fig. 7) and assuming that roughly half the variance (Wunsch 1997) is in the first baroclinic mode. The result is displayed in Fig. 8. Open ocean values are on the order of a few tenths of a degree, with far larger values in the regions of intense variability on the western sides of the ocean.

Ponte (2012) mapped the abyssal steric height changes from an eddy-permitting ECCO estimate, with patterns similar to those seen in Fig. 8, as one would expect from a model constrained to the altimetry. The space-time structure, that is, the mesoscale signal, is the noise background for abyssal trend determination, which will then depend upon the frequency-wavenumber spectrum of the altimetric variability. The problem is discussed elsewhere (Wunsch 2010).

\section{Energetics: The internal wave band}

For frequencies $f<\sigma<N$, thus omitting the betaplane complexities at and near the inertial frequency,

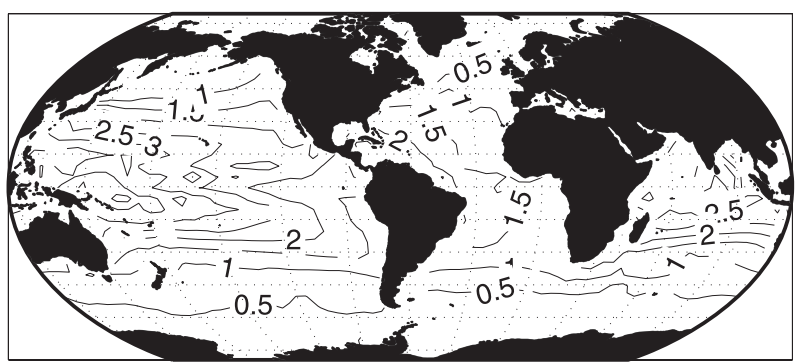

FIG. 5. The surface deflection corresponding to the eigenvalues in Fig. 4 in centimeters for a 10-m maximum isopycnal deflection within the water column. and the minor nonhydrostatic complications as $\sigma \rightarrow N$, the Cartesian $f$-plane version of Eq. (4) in $P$ is

$$
\nabla_{h}^{2} P+\frac{\sigma^{2}-f^{2}}{g h_{j}^{\prime}} P(x, y)=0,
$$

describing long internal waves with the meaning of $h_{j}^{\prime}$ made apparent: corresponding to the ordinary water depth of a constant density ocean, albeit of magnitude on the order of $1 \mathrm{~m}$. A large literature exists on the forced version of this limit, much of it through studies of sources sustaining the Garrett and Munk (1972) spectrum (e.g., Thorpe 2005). The much-studied internal tides with fixed frequencies span the complete range in this equation of $\sigma^{2}-f^{2}$, as the latitude changes, as well as depending upon the variations in $h_{j}^{\prime}$.

A number of authors (e.g., Nash et al. 2005) have discussed internal wave energetics. To give one example

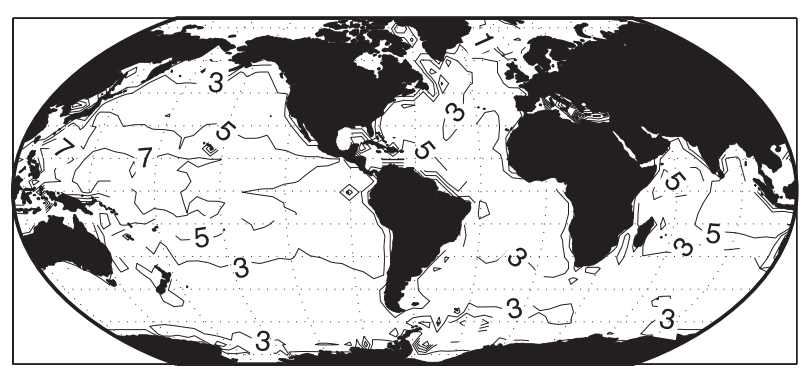

FIG. 6. Ratio of the horizontal current at the surface and bottom, $|F(0) / F(-h)|$, which is a simple measure of the surface intensification. The same ratio corresponds to that of surface and bottom pressure perturbations. 


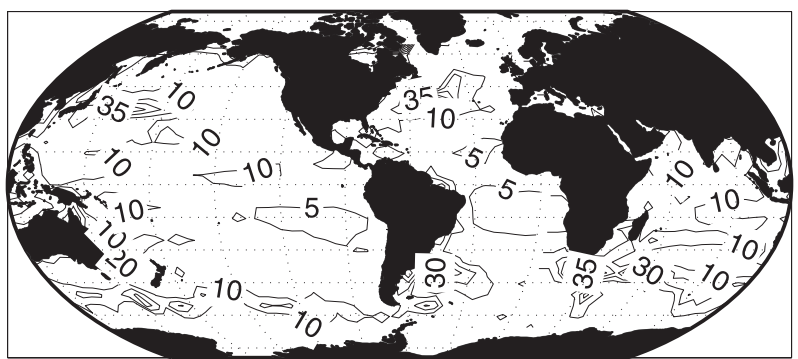

FIG. 7. RMS value of $\eta(\mathrm{cm})$ from the along-track TOPEX/ Poseidon-Jason altimetric data. Contouring is incomplete in the western boundary current maximum areas.

of the importance of the surface boundary condition values, consider the potential energy (PE) of an internal wave [Gill 1982, Eq. (6.7.13), inserting a missing integral sign]

$\mathrm{PE}=\frac{1}{4}\left[\rho_{0}(0) g \eta^{2}+B^{2} \int_{-h}^{0} \rho_{0} N(z)^{2} G_{D}(z)^{2} d z\right]$

in the present notation and where the horizontal spacetime dependence is a propagating exponential. Note that $\eta$ is also proportional to $B$. As in Gill (1982), the PE is made of two parts, one owing to the surface elevation and the other to the interior vertical displacements.

For simplicity, the present discussion is confined to the potential energy, because unlike the kinetic energy, it can be calculated from the vertical displacement alone and is not dependent upon the horizontal wavelengths (e.g., LeBlond and Mysak 1978; Phillips 1980; Gill 1982). Studies of, for example, the complete internal tide energetics from altimeters thus require calculations employing solutions to Eq. (15) or equivalent, and are beyond the present intentions of this paper (but see, e.g., Rainville and Pinkel 2006).

For realistic values of the parameters, the second term in Eq. (16) is about two orders of magnitude greater than the first. The second term declines by a factor of 3-5 from the tropics toward the poles for a fixed vertical displacement amplitude, $B$ (not shown). Thus a wave conserving potential energy while traveling poleward would have to increase its observed squared surface amplitude by a corresponding amount.

\section{a. Balanced motion band}

At the low-frequency limit, $\sigma \ll f$, there exist a few interesting issues, some of them arising because of the recent extended discussion of the so-called surface quasigeostrophic approximation. Here we only sketch this idea, roughly following the development of LaCasce (2012).

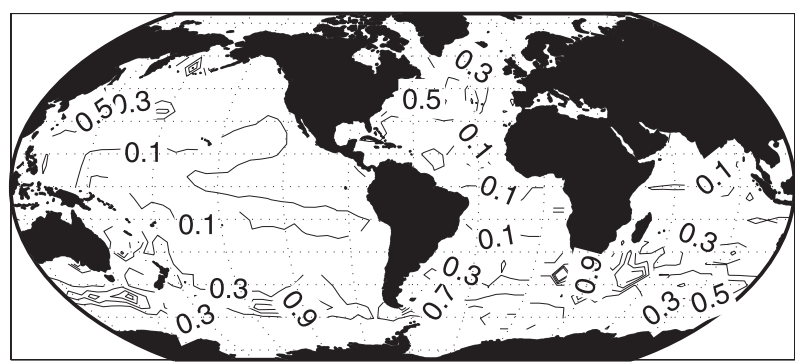

FIG. 8. RMS temperature perturbation $\left({ }^{\circ} \mathrm{C}\right)$ from the RMS altimetric values of $\eta$, at the depth of the maximum vertical displacement in $G_{\mathrm{D} 1}$. Contouring is incomplete in the boundary current regions. These values are an estimate of the noise, dominantly that of mesoscale eddies, that would appear in abyssal hydrography.

In this low-frequency limit where motions are nearly in quasigeostrophic balance, Eq. (4) reduces to the statement of linear conservation of potential vorticity (Pedlosky 1987, p. 367), and which is for $P$, after the separation of variables and the assumption of periodic motions,

$$
\nabla_{h}^{2} P+\frac{\beta}{i \sigma} \frac{\partial P}{\partial x}-\frac{f^{2}}{g h_{i}^{\prime}} P=0
$$

For free motions subject to the top and bottom boundary conditions analyzed above, no novel features appear [worked out again explicitly by Wunsch and Stammer (1997, hereafter WS) among other places].

In the conventional unforced SQG discussion (e.g., LaCasce 2012), the $\beta$ term is dropped and a new surface boundary condition is imposed in which the surface density anomaly, $\rho^{\prime}(x, y, z=0, \sigma)$, is regarded as known - and is to be used to infer the interior motions. ${ }^{2}$ Dropping $\beta$ implies that sufficiently small scales (submesoscale) motions are being discussed (failure of hydrostatic balance could become a concern). In the present context, its main consequence is that $h_{i}^{\prime}<0$, always. Retaining $\beta$ does not introduce a fundamentally new mathematics, although permitting ranges of positive $h_{i}^{\prime}$.

Density anomalies $\rho^{\prime}(x, y, z=0, \sigma)$ at the sea surface can be prescribed as long as there is no mixed layer, but the dynamical boundary condition at $z=0$ must still be satisfied. Let $\rho^{\prime}(x, y, z=0, \sigma)=A_{0} G_{D}(0) \exp (i k x+i l y-i \sigma t)$, and $P(x, y, \sigma)=B_{0} \exp (i k x+i l y-i \sigma t) . h_{i}^{\prime}\left(\right.$ or $\left.\gamma_{i}^{2}\right)$ is determined from Eq. (17). From Eq. (1d),

\footnotetext{
${ }^{2}$ The existence of surface density anomalies dependent upon temperature perturbations will induce an atmospheric interaction. Whether these motions should be regarded as truly unforced becomes a semantic matter.
} 


$$
B_{0}=\frac{A_{0} g}{\rho_{0} N(0)^{2}}
$$

By setting, arbitrarily, $G_{D}(0)=1, A_{0}$ fixes the magnitude of the free surface movement, and the dynamic boundary condition [Eq. (6)] requires

$$
\frac{d G_{D}(0)}{d z}=g \gamma^{2}
$$

and one can solve Eq. (2) for $G(z)$, for example. The magnitude of $P(x, y, \sigma)$ is set by $B_{0}$ and then Eq. (1b) produces

$$
\eta(x, y, \sigma)=\frac{w(0)}{-i \sigma}=\frac{A_{0} g}{\rho_{0} N(0)^{2}} \exp (i k x+i l y-i \sigma t) .
$$

These results will be susceptible to spatial variations in $N(0)$, and in particular, to the appearance of mixed layers, perhaps seasonally, and requiring care in the limit as $N(0) \rightarrow 0$ and thus influence inferences from altimetric data. In real mixed layers, there exist lateral gradients in density, but these preclude the use of the separation of variables approach.

The above results apply directly only to modes, that is, free waves-those satisfying homogeneous top and bottom boundary conditions. The more interesting situation is when the motion is forced, perhaps at the free surface, with $k, l$, and $\sigma$ set by the forcing. Then the separation constant $\gamma^{2}$ or its equivalent, $h_{i}^{\prime}$, is determined through the requirement that Eq. (17) must be satisfied. As various textbook discussions make clear, $h_{i}^{\prime}$ can become negative in certain ranges of $k, l$, and $\sigma$, rendering the corresponding solutions in the vertical to Eq. (2) or (3) exponential rather than sinusoidal. (But recall that conventionally, a "mode" is an unforced, dissipationless, free oscillation of a system with a characteristic frequency.) Forced linear responses bring a necessary attention to detail that has a long history, probably starting with the investigations of atmospheric load response in the nineteenth century (see the review by WS). Interest greatly increased after the discovery of the mesoscale, as described in papers by Philander (1978), Müller and Frankignoul (1981), and others. For the forced problem, Moore and Philander (1977) discuss the sensitivity of mode selection as a function of mixed layer depth. WS examine the sometimes intricate response to pressure loading, which moves the sea surface directly. Wind stress fluctuations at all frequencies will drive the dominant forced response, but that subject is not pursued here.

\section{b. Band confusion}

The focus in altimetric studies of the internal wave band has been on the internal tides (Ray and Mitchum 1997). Because of the sampling characteristics of altimetric missions, the principal lunar tide appears, for instance, in the TOPEX/Poseidon-Jason missions data as an alias near 60 days, with other tidal constituents distributed over the whole spectrum within the balanced band. In many records, however, the tidal bands are only a fraction, sometimes a small one, of the total energy lying in frequencies $f \leq \sigma \leq N$, and these will all alias into periods longer than 20 days, periods normally associated with the balanced motion band.

Current meter spectra in Ferrari and Wunsch (2009) and in other papers typically show semidiurnal tides having less than $50 \%$ of the internal wave kinetic energy, and sometimes much less. The extent to which aliased broadband internal wave energy is mistakenly attributed to balanced flows will depend upon the vertical scales (or mode numbers) of the ambient internal waves. It is possible, but not pursued here, that a significant fraction of the apparent energy being interpreted as geostrophic is aliased energy of the gravity wave band-and is a further reason for care in using measurements of $\eta$. Dynamical coupling of the bands, particularly through topographic interaction, is another large subject not taken up here.

\section{Discussion}

This study is incomplete in several respects. In particular, one must keep in mind that the spatial changes mapped here are likely to be quantitatively useful only in a WKBJ sense, with amplification factors changing slowly over the large regions depicted in the figures. Regions of rapid horizontal gradients that are apparent, but likely underestimated, in many of the figures will, in contrast, generate lateral structures of the type appearing in any wave motion encountering sharp index of refraction changes. None of those possibilities is explored here.

The near-surface structures of $N(z)$ will in practice undergo changes with season, over climate time scales, and over shorter periods depending upon the large-scale background oceanography and complex modulations from the eddy field. Very large seasonal changes in mixed layer depth do occur at high latitudes, and their quantitative effects on inferences there from altimetry have not been dealt with. [Seasonal cycle effects in the balanced limit were described by Wunsch (1997), and Ray and Zaron (2011, their Fig. 3) showed a seasonal phase change in the internal tide.]

If the altimetric variability is used to constrain realistic general circulation or internal tide models [as in Egbert 

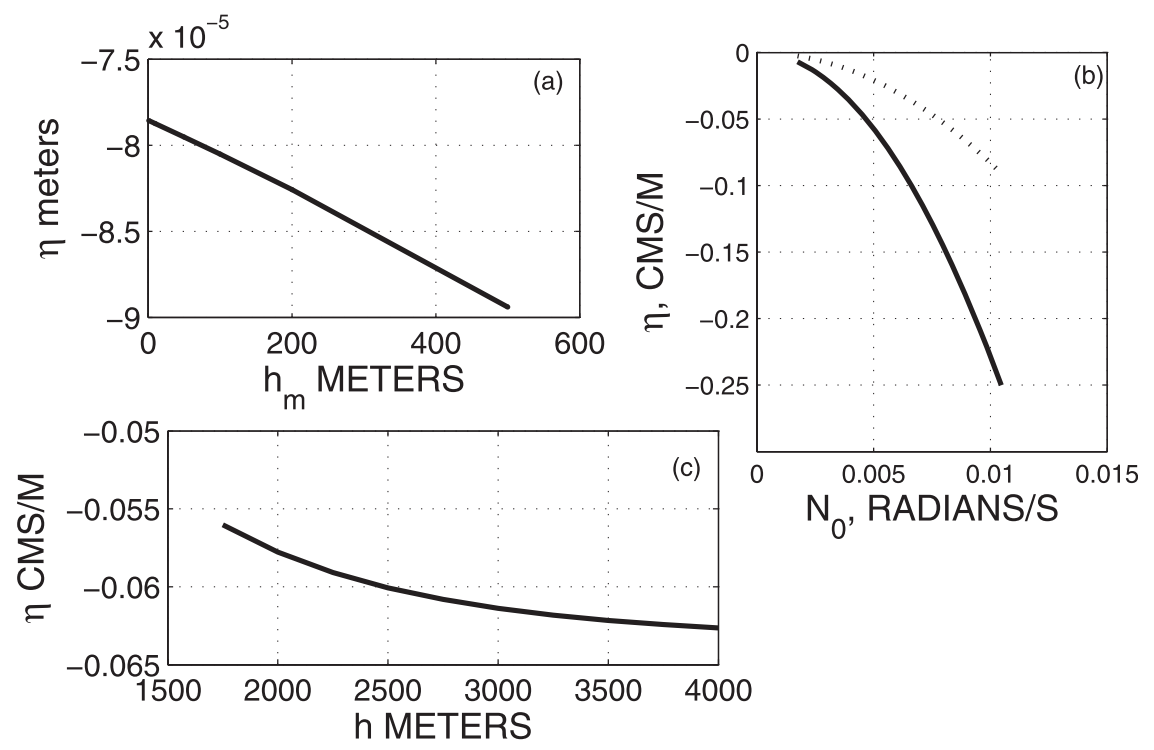

FIG. A1. (a) The quantity $\eta$ as a function of the mixed layer depth $h_{m}$ for a unit maximum interior vertical displacement and constant $N$. (b) The value of $\eta$ in centimeters for a $1-\mathrm{m}$ maximum vertical displacement in the interior as a function of $N_{0}$ in an ocean of depth $h=$ $4000 \mathrm{~m}$ for the exponential profile (solid curve). The result produces millimeters of elevation per meter of interior vertical displacement. Dotted line shows the same calculation but made using the rigid-lid boundary condition and the associated surface pressure field. (c) Dependence upon the relative amplitude of the free-surface displacement as a function of the water depth for an ocean with $N_{0}$ corresponding to a 20 -min period in the exponential profile.

and Ray (2003) for the latter and Wunsch et al. (2009) for the former], adaptation of the model to the space- and time-varying values of $N(z, x, y, t)$ and thus of the elevation $\eta$, is "automatic" and one need not resort to the modal structures as employed here. Such calculations are to be preferred, as they also account for the nonseparable effects of varying bottom topography, $h(x, y)$, as well as major complications including the presence of lowerfrequency "mean" current systems. On the other hand, the simple, approximate, modal description produces insight into the underlying physics, and its understanding is probably essential to interpreting the model results.

Acknowledgments. Supported in part by NASA Grant NNX08AR33G (altimetry). Prepared while I was George Eastman Visiting Professor, Balliol College and AOPP, University of Oxford. I had very helpful comments from R. Ray, R. Ponte, J. LaCasce, J. Callies, and the anonymous reviewers.

\section{APPENDIX}

\section{Vertical Modes}

\section{a. Mixed layer over constant $N$}

One can compute $\eta$ for an infinite number of analytic profiles, but the point of diminishing returns rapidly sets in. Gaining some understanding of the presence of a mixed layer is, however, useful. Lighthill (1969) and Gill (1975) considered a three-layer model, with $N(z=0),-h_{m} \leq z \leq 0, N=N_{0},-h \leq z \leq-h_{m}, N=$ $N_{0}$, and $-\infty \leq z \leq-h$-that is, a mixed layer overlying a uniformly stratified layer, in turn overlying an infinitely deep unstratified one. In the infinitely thick layer, horizontal velocities become vanishingly small and one has a slightly simplified problem from removal of the bottom boundary condition. Here, we impose the rigid boundary condition at $z=-h$, leading to the values of $\eta$ as a function of $h_{m}$ shown in Fig. A1a.

In this situation, competition exists between the presence of a mixed layer, which reduces the amount of vertically displaced heavy water when $w>0$, thus reducing the value of $\eta$ required for compensation, and the deepening of the position where $w$ reaches its maximum value (at the level of no horizontal motion) and which increases the mass of denser water (Fig. A2). In this parameter range, the deepening effect is larger, and $\eta$ increases slowly with the mixed layer depth.

\section{b. Exponential $N(z)$}

Constant $N$ is too Procrustean a representation to be applied easily to the real ocean. A useful compromise 
(a)

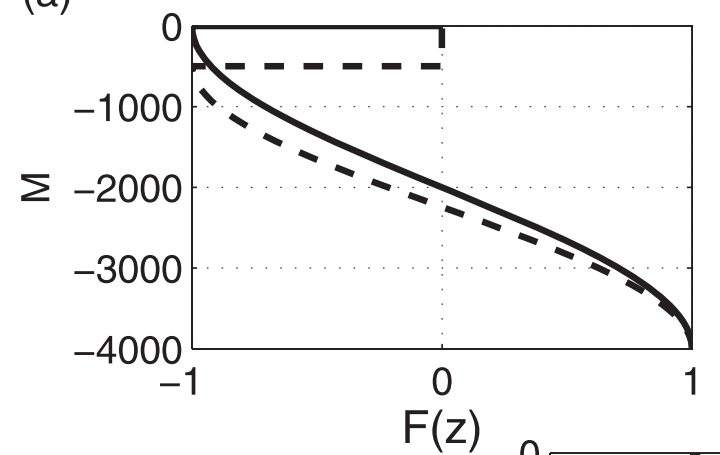

(b)

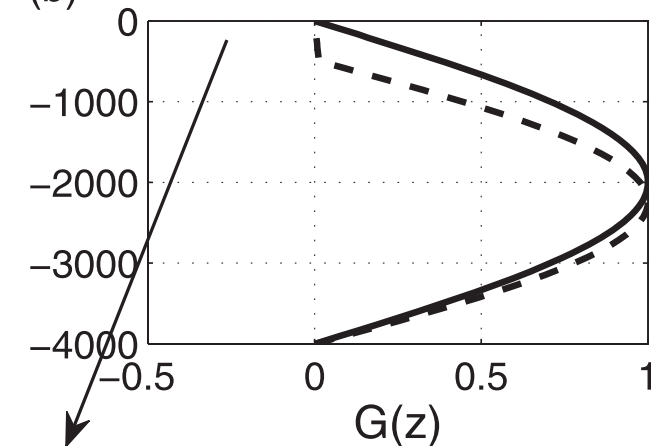

(c)

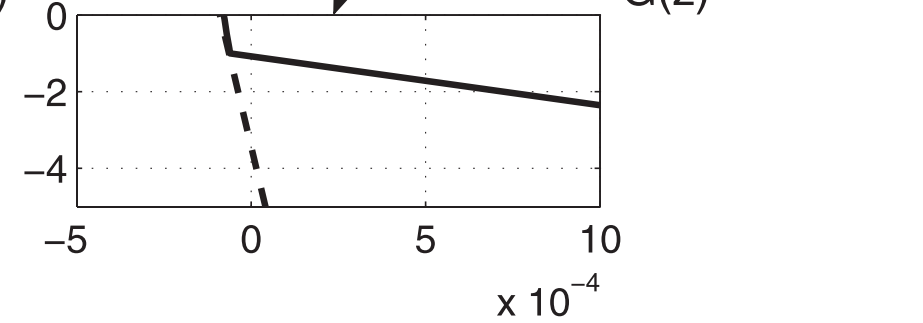

FIG. A2. Values of (a) $F_{1}$ and (b) $G_{\mathrm{D} 1}$ in an ocean of constant $N$ with $h=4000 \mathrm{~m}$, overlaid by a mixed layer of $500 \mathrm{~m}$ (dashed lines) as compared to one that is exponential everywhere (solid lines), showing (a) horizontal and (b) vertical displacements. (c) The overshoot in the vertical displacement in the top $5 \mathrm{~m}$.

between a fully realistic profile and the constant one is the exponential

$$
N(z)=N_{0} e^{\lambda z}
$$

which has been widely used, for example by Garrett and Munk (1972), WG, and many others. WG used the profile to explain the occurrence in tropical tide gauge data of internal wave branch first baroclinic mode equatorially trapped waves and we take that calculation as a starting point. Solutions to Eq. (2) are now

$$
G(z)=A_{1} J_{0}\left(\frac{\gamma N_{0}}{\lambda} e^{\lambda z}\right)+A_{2} Y_{0}\left(\frac{\gamma N_{0}}{\lambda} e^{\lambda z}\right)
$$

where $J_{0}$ and $Y_{0}$ are the Bessel functions.

WG set the effective lower boundary at $z=-\infty$ and put $A_{2}=0$ to prevent an exponential singularity in $Y_{0}$. Equation (6) then requires

$$
J_{1}\left(\frac{N_{0} \gamma}{\lambda}\right)-\frac{g \gamma}{N_{0}} J_{0}\left(\frac{N_{0} \gamma}{\lambda}\right)=0
$$

exploiting the identity $J_{0}^{\prime}=-J_{1}$, and which can be solved numerically for $\gamma$ (this equation is misprinted in WG).

With an infinitely deep ocean, the use of the $J_{\nu}$ Bessel functions can be questioned, as they represent standing wave solutions. One might suspect that the downward radiating Hankel function, $H_{0}^{(1)}\left[\left(\gamma N_{0} / \lambda\right) e^{\lambda z}\right]$ is more appropriate. A standing wave solution is possible in this situation, even with infinite depth, because $N(z)$ ultimately becomes smaller than any possible frequency, $\sigma$, trapping the waves between the depth where $\sigma=N(z)$ and the sea surface. Although using a finite depth makes little change to the surface motions, the WG calculation is here reformulated with a finite value of $h$, retaining both Bessel functions.

The bottom boundary condition is

$$
A_{1} J_{0}\left(\Delta e^{-\lambda h}\right)+A_{2} Y_{0}\left(\Delta e^{-\lambda h}\right)=0,
$$

with $\Delta=\gamma N_{0} / \lambda$, and the surface one is

$$
\frac{g \gamma}{N_{0}}\left[A_{1} J_{0}(\Delta)+A_{2} Y_{0}(\Delta)\right]-\left[A_{1} J_{0}^{\prime}(\Delta)+A_{2} Y_{0}^{\prime}(\Delta)\right]=0
$$

for two equations whose vanishing determinant is solved for $\Delta$ and $\gamma$. The vertical displacement is, for the first vertical mode,

$$
\begin{aligned}
G_{1}(z)= & A\left\{J_{0}\left(\frac{N_{0} \gamma_{1}}{\lambda} e^{\lambda z}\right)-\frac{J_{0}\left[\left(N_{0} \gamma_{1} / \lambda\right) e^{-\lambda h}\right]}{Y_{0}\left[\left(N_{0} \gamma_{1} / \lambda\right) e^{-\lambda h}\right]}\right. \\
& \left.\times Y_{0}\left(\frac{N_{0} \gamma_{1}}{\lambda} e^{\lambda z}\right)\right\},
\end{aligned}
$$

using Eq. (23), with surface displacement again $\eta=$ $G_{\mathrm{D} 1}(0)$. Choosing $A$ so that the maximum value of $G_{\mathrm{D} 1}$ 
in the water column is $1 \mathrm{~m}$, Fig. $9 \mathrm{~b}$ displays the value of $\eta$ as a function of $N_{0}$ in an ocean with $h=4000 \mathrm{~m}$. Thus a 10-m vertical displacement within the water column will move the sea surface by about $1-2 \mathrm{~cm}$, consistent with the WG value. The solution exhibits a strong dependence upon $N_{0}$, particularly for high values of $N_{0}$. Figure $9 \mathrm{~b}$ also displays the results obtained by computing $\eta$ from the rigid-lid pressure, as discussed in the main text.

Figure $9 \mathrm{c}$ shows the dependence of $\eta$ on $h$ for an ocean with a fixed value of $N_{0}=0.0052 \mathrm{~s}^{-1}$ (about a 20-min period). As the water shoals, the column average value of $N(z)$ increases, and thus the product $\bar{N} h$ is to a degree self-compensating. Fennel and Lass (1989) formulate the problem of a mixed layer over an exponential $N(z)$, but no new phenomena appear.

\section{REFERENCES}

Bobrovich, A. V., and G. M. Reznik, 1999: Planetary waves in a stratified ocean of variable depth. Part 2. Continuously stratified ocean. J. Fluid Mech., 388, 147-169.

Chiswell, S. M., 2006: Altimeter and current meter observations of internal tides: Do they agree? J. Phys. Oceanogr., 36, 1860-1872.

Egbert, G. D., and R. D. Ray, 2003: Semi-diurnal and diurnal tidal dissipation from TOPEX/Poseidon altimetry. Geophys. Res. Lett., 30, 1907, doi:10.1029/2003GL017676.

Fennel, W., and H. U. Lass, 1989: Analytical Theory of Forced Oceanic Waves. Academie Verlag, 312 pp.

Ferrari, R., and C. Wunsch, 2009: Ocean circulation kinetic energy: Reservoirs, sources, and sinks. Ann. Rev. Fluid Mech., 41, 253282.

Garrett, C. J. R., and W. H. Munk, 1972: Space-time scales of internal waves. Geophys. Fluid Dyn., 3, 225-264.

Gill, A. E., 1975: Models of equatorial currents. Numerical Models of Ocean Circulation, National Academies of Sciences Press, 181-203.

— 1982: Atmosphere-Ocean Dynamics. Academic Press, 662 pp.

Isern-Fontanet, J., G. Lapeyre, P. Klein, B. Chapron, and M. W. Hecht, 2008: Three-dimensional reconstruction of oceanic mesoscale currents from surface information. J. Geophys. Res., 113, C09005, doi:10.1029/2007JC004692.

LaCasce, J. H., 2011: Surface quasi-geostrophic solutions and baroclinic modes with exponential stratification. J. Phys. Oceanogr., 42, 569-580.

—_, and A. Mahadevan, 2006: Estimating subsurface horizontal and vertical velocities from sea-surface temperature. J. Mar. Res., 64, 695-721.

LeBlond, P. H., and L. Mysak, 1978: Waves in the Ocean. Elsevier, $602 \mathrm{pp}$.
Lighthill, M. J., 1969: Dynamic response of Indian Ocean to onset of the southwest monsoon. Philos. Trans. Roy. Soc. London, A265, 45-92.

Moore, D. W., and S. G. H. Philander, 1977: Modeling of the tropical oceanic circulation. The Sea-Ideas and Observations on Progress in the Study of the Seas, E. D. Goldberg et al., Eds., Vol. 6, Wiley-Interscience, 319-361.

Müller, P., and C. Frankignoul, 1981: Direct atmospheric forcing of geostrophic eddies. J. Phys. Oceanogr., 11, 287-308.

Nash, J. D., M. H. Alford, and E. Kunze, 2005: Estimating internal wave energy fluxes in the ocean. J. Atmos. Oceanic Technol., 22, 1551-1570.

Pedlosky, J., 1987: Geophysical Fluid Dynamics. 2nd ed. SpringerVerlag, 710 pp.

Philander, S. G. H., 1978: Forced oceanic waves. Rev. Geophys., 16, $15-46$.

Phillips, O. M., 1980: The Dynamics of the Upper Ocean. 2nd ed. Cambridge University Press, $336 \mathrm{pp}$.

Ponte, R. M., 2012: An assessment of deep steric height variability over the global ocean. Geophys. Res. Lett., 39, L04601, doi:10.1029/2011gl050681.

Rainville, L., and R. Pinkel, 2006: Propagation of low-mode internal waves through the ocean. J. Phys. Oceanogr., 36, 1220-1236.

Ray, R. D., and G. T. Mitchum, 1997: Surface manifestation of internal tides in the deep ocean: Observations from altimetry and island gauges. Prog. Oceanogr., 40, 135-162.

— served with satellite altimetry. Geophys. Res. Lett., 38, L17609, doi:10.1029/2011GL048617.

Thorpe, S. A., 2005: The Turbulent Ocean. Cambridge University Press, $439 \mathrm{pp}$.

Wortham, C., 2012: A multi-dimensional spectral description of oceanic variability with applications. Ph.D. thesis, MIT/ WHOI Joint Program in Physical Oceanography, 184 pp.

Wunsch, C., 1997: The vertical partition of oceanic horizontal kinetic energy. J. Phys. Oceanogr., 27, 1770-1794.

_ 2010: Towards a midlatitude ocean frequency-wavenumber spectral density and trend determination. J. Phys. Oceanogr., 40, 2264-2281.

_ , and A. E. Gill, 1976: Observations of equatorially trapped waves in Pacific sea level variations. Deep-Sea Res., 23, 371390.

_ and D. Stammer, 1997: Atmospheric loading and the oceanic "inverted barometer" effect. Rev. Geophys., 35, 79-107.

— , and Coauthors, 2009: The global general circulation of the ocean estimated by the ECCO-Consortium. Oceanography, 22, 88-103.

Xu, C., X.-D. Shang, and R. Huang, 2011: Estimate of eddy energy generation/dissipation rate in the world ocean from altimetry data. Ocean Dyn., 61, 525-541.

Zhao, Z. X., M. H. Alford, J. A. MacKinnon, and R. Pinkel, 2010: Long-range propagation of the semidiurnal internal tide from the Hawaiian Ridge. J. Phys. Oceanogr., 40, 713-736. 